\title{
IMPROVEMENT TO ESPRIT-TYPE FREQUENCY ESTIMATORS VIA REDUCING DATA REDUNDANCY
}

\author{
Weize Sun and H.C. So \\ Department of Electronic Engineering, City University of Hong Kong, Hong Kong SAR, China
}

\begin{abstract}
In this paper, the problem of estimating the damping factor and frequency parameters from multiple cisoids in noise is addressed. We first propose a data matrix which generalizes the commonly used Hankel-style matrices so that the number of repeated entries can be reduced. A new computationally efficient ESPRIT estimator that makes use of the right singular vectors is then devised. Algorithm modification for undamped sinusoids and complexity are also discussed. Computer simulations are included to compare the proposed approach with the conventional ESPRIT methods and CramérRao lower bound.
\end{abstract}

Index Terms - frequency estimation, subspace method, singular value decomposition

\section{INTRODUCTION}

The topic of sinusoidal parameter estimation from a finite number of noisy discrete-time measurements has attracted a great deal of attention because of its wide applications in science and engineering. Basically, estimating the damping factor and frequency parameters is the crucial step because they are non-linear functions in the observed data, and computation of the remaining parameters can then reduce to a least squares (LS) fit.

Generally speaking, spectral estimation can be achieved by means of either nonparametric or parametric approaches [1]. The nonparametric methods are simply based on the Fourier transform but their ability to resolve closely-spaced frequencies is fundamentally limited by the length of the data available. On the other hand, the parametric approach, which assumes that the signal satisfies a generating model with known functional form, can attain a higher resolution. Among the parametric techniques, the subspace methods such as multiple signal classification (MUSIC) [2] and estimation of signal parameters via rotational invariance techniques (ESPRIT) [3] have received considerable interest because they are attractive alternatives to maximum-likelihood based methodology which requires extensive computations. Their underlying principle is to separate the data into signal and

The work described in this paper was supported by a grant from CityU (Project no. 7002570). noise subspaces via eigenvalue decomposition of the sample covariance matrix or the singular value decomposition (SVD) of the raw data matrix. Conventionally, a Hankelstyle matrix is constructed for parameter estimation, which implies a large data redundancy. In this work, we propose to reduce the data reuse and employ the ESPRIT methodology as an illustration. Although our primary objective is to achieve computation reduction, the proposed estimator can be considered a generalized subspace method.

The rest of this paper is organized as follows. The problem formulation for sinusoidal parameter estimation is given in Section 2. The proposed estimator for damping factor and frequency parameters are derived in Section 3. Its comparative computational complexity and forward-backward averaging modification for undamped cisoids are also provided. In Section 4, simulation results are included to evaluate the performance of the proposed approach by comparing with the ESPRIT algorithms [4] as well as Cramér-Rao lower bound (CRLB). Finally, conclusions are drawn in Section 5.

\section{PROBLEM STATEMENT}

Throughout this paper, bold upper case symbols denote matrices, and bold lower case symbols denote column vectors. We denote $N_{1} \times N_{2}$ zero matrix, $N \times N$ identity matrix and $N \times N$ matrix with ones in its anti-diagonal and zeros elsewhere, by $\mathbf{0}_{N_{1} \times N_{2}}, \mathbf{I}_{N}$ and $\boldsymbol{\Pi}_{N}$, respectively. The Kronecker product and the 1-norm operator are represented by $\otimes$ and \|\|$_{1}$, and superscripts ${ }^{T},{ }^{H},{ }^{*},{ }^{-1}$ and ${ }^{\dagger}$ denote transpose, Hermitian transpose, complex conjugation, matrix inversion and pseudo-inverse, respectively. Moreover, we use $\tilde{\mathbf{A}}$ and $\hat{\mathbf{A}}$ to represent the noise-free counterpart and estimate of $\mathbf{A}$.

The observed noisy sinusoidal signal is modeled as:

$$
\begin{aligned}
x_{n} & =s_{n}+\xi_{n}, \quad n=1,2, \cdots, N \\
\text { where } & s_{n}=\sum_{k=1}^{K} \gamma_{k} \alpha_{k}^{n} e^{i\left(\omega_{k} n+\varphi_{k}\right)}, \quad k=1,2, \cdots, K
\end{aligned}
$$

The $\gamma_{k}>0, \alpha_{k} \in(0,1], \omega_{k} \in[-\pi, \pi)$ and $\varphi_{k} \in[-\pi, \pi)$ are the sinusoidal amplitudes, damping factors, frequencies and phases while $\left\{\xi_{n}\right\}$ are white complex Gaussian noises of zero mean and unknown variance $\sigma^{2}$. The number of sinusoids, denoted by $K$, is assumed known a priori. 
We use a matrix $\mathbf{X} \in \mathbb{C}^{N_{1} \times N_{2}}$ to represent (1), which has the form of:

$$
\mathbf{X}=\mathbf{S}+\boldsymbol{\Xi}
$$

where

$$
\begin{aligned}
\mathbf{S} & =\left[\begin{array}{llll}
\mathbf{s}_{1} & \mathbf{s}_{2} & \cdots & \mathbf{s}_{N_{2}}
\end{array}\right] \\
\mathbf{s}_{n_{2}} & =\left[\begin{array}{llll}
s_{\left(n_{2}-1\right) M+1} & s_{\left(n_{2}-1\right) M+2} & \cdots & s_{\left(n_{2}-1\right) M+N 1}
\end{array}\right]^{T} \\
n_{2} & =1,2, \cdots, N_{2}
\end{aligned}
$$

The $\Xi$ contains $\left\{\xi_{n}\right\}$ accordingly and $M \in\left[1, N_{1}\right]$ is an integer which controls the data reuse. With proper selection of integers $M, N_{1}$ and $N_{2},\left(N_{2}-1\right) M+N_{1}=N$ is satisfied, which means that all elements of $\left\{x_{n}\right\}$ in (3) appear at least once. When $M=1, \mathbf{X}$ corresponds to a Hankel matrix which involves the maximum number of repeated entries. On the other hand, there is no repeated element when assigning $M=N_{1}$. As a result, $\mathbf{X}$ can be considered as a generalized data matrix for the subspace methodology.

\section{ALGORITHM DEVELOPMENT}

\subsection{Proposed Estimator}

Following [4]-[5], S can be factorized as:

$$
\begin{aligned}
& \mathbf{S}=\mathbf{G} \boldsymbol{\Gamma} \mathbf{H}^{T} \\
& \text { where } \boldsymbol{\Gamma} \\
&=\operatorname{diag}\left(\gamma_{1} e^{i \varphi_{1}}, \gamma_{2} e^{i \varphi_{2}}, \cdots, \gamma_{K} e^{i \varphi_{K}}\right) \\
& \mathbf{G}=\left[\begin{array}{llll}
\mathbf{g}_{1} & \mathbf{g}_{2} & \cdots & \mathbf{g}_{K}
\end{array}\right] \\
& \mathbf{H}=\left[\begin{array}{llll}
\mathbf{h}_{1} & \mathbf{h}_{2} & \cdots & \mathbf{h}_{K}
\end{array}\right] \\
& \mathbf{g}_{k}=\left[\begin{array}{llll}
g_{k} & g_{k}^{2} & \cdots g_{k}^{M}
\end{array}\right]^{T} \\
& \mathbf{h}_{k}=\left[\begin{array}{lll}
h_{k} & h_{k}^{2} & \cdots h_{k}^{N}
\end{array}\right]^{T} \\
& g_{k}=\alpha_{L, k} e^{i \omega_{L, k}} \quad \text { and } h_{k}=\beta_{k} e^{i \mu_{k}}
\end{aligned}
$$

Apparently, we have $\alpha_{L, k}=\alpha_{k}, \omega_{L, k}=\omega_{k}, \beta_{k}=\alpha_{k}^{M}$ and $\mu_{k}=\left(M \omega_{k}\right) \bmod (2 \pi)$ for all $k$. On the other hand, decomposing $\mathbf{X}$ using SVD gives:

$$
\mathbf{X}=\mathbf{U} \boldsymbol{\Lambda} \mathbf{V}^{H}=\left[\begin{array}{ll}
\mathbf{U}_{s} & \mathbf{U}_{n}
\end{array}\right]\left[\begin{array}{cc}
\boldsymbol{\Lambda}_{s} & \mathbf{0} \\
\mathbf{0} & \boldsymbol{\Lambda}_{n}
\end{array}\right]\left[\begin{array}{ll}
\mathbf{V}_{s} & \mathbf{V}_{n}
\end{array}\right]^{H}
$$

where $\mathbf{U}_{s} \in \mathbb{C}^{N_{1} \times K}, \boldsymbol{\Lambda}_{s} \in \mathbb{C}^{K \times K}$ and $\mathbf{V}_{s} \in \mathbb{C}^{N_{2} \times K}$ are the signal subspaces. According to the decomposition in (5)(11), the best rank- $K$ approximation of $\mathbf{S}$ according to (12), denoted by $\hat{\mathbf{S}}$, is

$$
\hat{\mathbf{S}}=\mathbf{U}_{s} \boldsymbol{\Lambda}_{s} \mathbf{V}_{s}^{H}
$$

According to [6], define the selection matrices $\mathbf{J}_{U 1}=$ $\left[\begin{array}{ll}\mathbf{I}_{N_{1}-1} & \mathbf{0}_{\left(N_{1}-1\right) \times 1}\end{array}\right]$ and $\mathbf{J}_{U 2}=\Pi_{N_{1}-1} \mathbf{J}_{U 1} \Pi_{N_{1}}$, the estimates of $g_{k}$ 's, denoted by $\hat{g}_{k}$ 's, can be retrieved from the $K$ eigenvalues of $\boldsymbol{\Psi}_{U}$ :

$$
\mathbf{\Psi}_{U}=\left(\mathbf{J}_{U 1} \mathbf{U}_{s}\right)^{\dagger} \mathbf{J}_{U 2} \mathbf{U}_{s}=\mathbf{T}_{U}^{-1} \mathbf{F}_{U} \mathbf{T}_{U}
$$

Once $\hat{g}_{k}$ is obtained, the corresponding frequency and damping factor estimates are straightforwardly computed as

$$
\hat{\omega}_{L, k}=\angle\left(\hat{g}_{k}\right) \quad \text { and } \quad \hat{\alpha}_{L, k}=\left|\hat{g}_{k}\right|
$$

Conventional subspace schemes set $M=1$ and employ (14)-(15) [6] or the left singular vectors to estimate the parameters [7]. In this work, we exploit the right singular vectors for parameter estimation. Comparing (5) and (12), we apply the same technique to $\mathbf{V}_{s}$ :

$$
\boldsymbol{\Psi}_{V}=\left(\mathbf{J}_{V 1} \mathbf{V}_{s}^{*}\right)^{\dagger} \mathbf{J}_{V 2} \mathbf{V}_{s}^{*}=\mathbf{T}_{V}^{-1} \mathbf{F}_{V} \mathbf{T}_{V}
$$

where $\mathbf{J}_{V 1}=\left[\begin{array}{ll}\mathbf{I}_{N_{2}-1} & \mathbf{0}_{\left(N_{2}-1\right) \times 1}\end{array}\right]$ and $\mathbf{J}_{V 2}=\Pi_{N_{2}-1} \mathbf{J}_{V 1} \Pi_{N_{2}}$. Then the estimates $\hat{h}_{k}$ can be retrieved from the eigenvalues of $\boldsymbol{\Psi}_{V}$ and

$$
\hat{\mu}_{k}=\angle\left(\hat{h}_{k}\right) \quad \text { and } \quad \hat{\beta}_{k}=\left|\hat{h}_{k}\right|
$$

According to (4), we have $\tilde{h}_{k}=\tilde{g}_{k}^{M}$. Let $\hat{\mathbf{g}}_{U}$ and $\hat{\mathbf{h}}_{V}$ be the estimated eigenvalue vectors of $\boldsymbol{\Psi}_{U}$ and $\boldsymbol{\Psi}_{V}$ containing all the $\hat{h}_{k}$ 's and $\hat{g}_{k}$ 's in an unpaired manner. Define $\hat{\mathbf{G}}_{U}=$ $\left[\begin{array}{llll}\hat{\mathbf{g}}_{U, 1} & \hat{\mathbf{g}}_{U, 2} & \cdots & \hat{\mathbf{g}}_{U, K !}\end{array}\right]$ where $\hat{\mathbf{G}}_{U} \in \mathbb{C}^{K \times K !}$ contains the all permutations of $\hat{\mathbf{g}}_{U}$ arranged one-by-one in rows, it is clear that $\tilde{\mathbf{G}}_{U}^{M}$ also corresponds to all permutations of $\tilde{\mathbf{h}}_{V}$. We compute new $\hat{\mathbf{g}}_{U}=\hat{\mathbf{g}}_{U, f}$ where the index $f$ is determined as

$$
f=\arg \min _{i=1,2, \cdots, K !}\left\{\left\|\hat{\mathbf{h}}-\hat{\mathbf{g}}_{U, i}^{M}\right\|_{1}\right\}
$$

Then we get $\hat{\mathbf{g}}_{U}=\left[\begin{array}{llll}\hat{g}_{1} & \hat{g}_{2} & \cdots & \hat{g}_{K}\end{array}\right]^{T}$ and $\hat{\mathbf{h}}_{V}=$ $\left[\begin{array}{llll}\hat{h}_{1} & \hat{h}_{2} & \cdots & \hat{h}_{K}\end{array}\right]^{T}$ with elements $\hat{g}_{k}$ and $\hat{h}_{k}$ correctly paired-up when the signal-to-noise ratio (SNR) is sufficiently large. Substituting $\hat{g}_{k}$ 's and $\hat{h}_{k}$ 's into (15) and (17), the correct sets of $\left\{\hat{\omega}_{l, k}, \hat{\alpha}_{l, k}, \hat{\mu}_{k}, \hat{\beta}_{k}\right\}$ are obtained.

To estimate $\omega_{k}$ using $\mu_{k}$, we notice that $\hat{\mu}_{k}$ corresponds to $2\lfloor M / 2\rfloor+1$ possible estimates of $\omega_{k}$, where \lfloor\rfloor rounds the value to the nearest integer towards $-\infty$, denoted by $\hat{\omega}_{R, k, i}$, $i=-\lfloor M / 2\rfloor,-\lfloor M / 2\rfloor+1, \cdots,\lfloor M / 2\rfloor$ :

$$
\hat{\omega}_{R, k, i}=\frac{\hat{\mu}_{k}+2 \pi i}{M}
$$

We obtain $\hat{\omega}_{R, k}=\hat{\omega}_{R, k, f}$ where $f$ is computed from

$$
f=\arg \min _{i \in\{-\lfloor M / 2\rfloor,-\lfloor M / 2\rfloor+1, \cdots,\lfloor M / 2\rfloor\}}\left|\hat{\omega}_{R, i}-\hat{\omega}_{L}\right|
$$

And it is straightforward to see that

$$
\hat{\alpha}_{R, k}=\hat{\beta}_{k}^{1 / M}
$$

As it is shown in [5] that the accuracy of $\hat{\omega}_{R, k}$ and $\hat{\alpha}_{R, k}$ is much higher than that of $\hat{\omega}_{L, k}$ and $\hat{\alpha}_{L, k}$ when $K=1$ and $M=N_{1}$, we assign

$$
\hat{\omega}_{k} \approx \hat{\omega}_{R, k} \quad \text { and } \quad \hat{\alpha}_{k} \approx \hat{\alpha}_{R, k}
$$

as the final estimates.

\subsection{Complexity Analysis}

To compare the computational complexity of the proposed method and ESPRIT-type methods, we need to know the SVD complexity. An efficient solution among a large variety of SVD computation methods is the orthogonal iteration approach [8] which truncates a $N_{1} \times N_{2}$ matrix to rank $K$ 
using $k_{t} N_{1} N_{2} K$ multiplications, where $k_{t}$ is an algorithm dependent factor. For integers $M, N_{1}, N_{2}$ that satisfies $\left(N_{2}-1\right) M+N_{1}=N$ and defining $p=\frac{N_{1}}{N_{1}+N_{2}} \in(0,1)$, we have

$$
f(M, p)=\frac{(N-M)^{2} p(1-p)}{[M(1-p)+p]^{2}}
$$

multiplications for the SVD computation in (12). Apparently, the computation complexity decreases as $M$ increases.

\subsection{Modification for Undamped Cisoids}

When the all damping factors are equal to one, we employ the forward-backward technique to improve the proposed estimator as follows. Define

$$
\mathbf{Z}=\left[\begin{array}{c}
\mathbf{X} \\
\boldsymbol{\Pi}_{N_{1}} \mathbf{X}^{*} \boldsymbol{\Pi}_{N_{2}}
\end{array}\right]
$$

and substituting $\mathbf{Z}$ for $\mathbf{X}$ in (12), we get the new $\mathbf{U}_{s} \in$ $\mathbb{C}^{2 N_{1} \times K}$. Applying the same technique as in Section (3.1), the LS estimate of $g_{k}$ is obtained from the eigenvalues of

$$
\mathbf{\Psi}_{U}=\left(\left(\mathbf{I}_{2} \otimes \mathbf{J}_{U 1}\right) \mathbf{U}_{s}\right)^{\dagger}\left(\mathbf{I}_{2} \otimes \mathbf{J}_{U 2}\right) \mathbf{U}_{s}
$$

Following (15)-(22), $\hat{\omega}_{k}$ are obtained for the undamped scenarios.

\section{SIMULATION RESULTS}

Computer simulations have been carried out to evaluate the performance of the proposed algorithm for multiple damped and undamped sinusoids in the presence of white Gaussian noise. The average mean square error (MSE) is assigned to evaluate the algorithm performance. All results provided are averages of 1000 independent runs.

The first test mainly focuses on the different combinations of $N_{1}$ and $N_{2}$ by studying the average MSE for frequencies and damping factors versus $p$ under SNR $=30 \mathrm{~dB}$. The parameters settings are $N=100,\left(\omega_{1}, \omega_{2}, \omega_{3}\right)=$ $(0.1,0.2,0.3) \pi,\left(\alpha_{1}, \alpha_{2}, \alpha_{3}\right)=(0.99,0.98,0.97),\left(\gamma_{1}, \gamma_{2}, \gamma_{3}\right)=$ $(1,2,3)$, and $\left(\varphi_{1}, \varphi_{2}, \varphi_{3}\right)$ are uniformly selected between $[-\pi, \pi)$ in each independent trial. The 'SE' denotes the standard ESPRIT method in [6]. Figures 1 and 2 show that for different $M$, although the performance of the proposed estimator varies with different combination of $N_{1}$ and $N_{2}$, it gives a high accuracy if $p$ is appropriately chosen. Roughly speaking, it is proposed to choose a combination of $M, N_{1}$ and $N_{2}$ such that $p \approx 0.7$.

In the second test, the main focus is to study the performance of the algorithm under differen $M$. The parameters are the same as in the first test and we set $p \approx 0.7$ for the proposed approaches. The average computation times of the proposed method with $M=2,4,8$ as well as SE scheme are measured as $1 \times 10^{-3} \mathrm{~s}, 6.5 \times 10^{-4} \mathrm{~s}, 4.3 \times 10^{-4} \mathrm{~s}$ and $2.1 \times 10^{-3} \mathrm{~s}$, respectively. It is shown in figures 3 and 4 that the proposed estimator performs as well as ESPRIT with $M=2$ and 4 but a little worse in the situation $M=8$. The trade off between computation complexity and the estimation accuracy is clearly shown in this test.

In figures 5 and 6, we deal with the undamped sinusoids employing the forward-backward approach for different data length. 'UE' stands for 'Unitary ESPRIT' [6] as the forward-backward approach ESPRIT type method. The frequencies in figure 5 are $\left(\omega_{1}, \omega_{2}, \omega_{3}\right)=(0.1,0.2,0.3) \pi$ while $\left(\omega_{1}, \omega_{2}, \omega_{3}\right)=(0.1,0.13,0.16) \pi$ in figure 6 , which indicates a set of close frequencies. All other parameters are the same as previous except the data length $N$, and we set $M=2$ and $p_{2}=\frac{2 N_{1}}{2 N_{1}+N_{2}} \approx 0.7$ for the forward-backward approach. Both figures 5 and 6 show that the proposed forward-backward approach performs very well.

\section{CONCLUSION}

The aim of this paper is to design an algorithm makes benefits from the matrix structure of a general overlapped form for matrix (4) under integer $M \in\left[1, N_{1}\right]$, especially $M \geq 2$. Computer simulations show that by taking the advantages of the data structure of (4), the proposed estimator can achieve the same performance for $M>1$ as ESPRIT type algorithms $(M=1)$, with additional benefit of reduced complexity.

\section{REFERENCES}

[1] P. Stoica and R. Moses, Spectral Analysis of Signals, Upper Saddle River, NJ: Prentice-Hall, 2005

[2] R. Schmidt, "Multiple emitter location and signal parameter estimation," IEEE Transactions on Antennas and Propagation, vol.34, no.3, pp.276-280, Mar. 1986

[3] R. Roy, A. Paulraj and T. Kailath, "ESPRIT-A subspace rotation approach to estimation of parameters of cisoids in noise," IEEE Transactions on Acoustics, Speech and Signal Processing, vol.34, no.5, pp.1340-1342, Oct. 1986

[4] H.C. So, F.K.W. Chan, W.H. Lau and C.F. Chan, "An efficient approach for two-dimensional parameter estimation of a single-tone," IEEE Transactions on Signal Processing, vol.58, no.4, pp.1999-2009, Apr. 2010

[5] H.C. So, F.K.W. Chan, W. Sun, "Subspace approach for fast and accurate single-tone frequency estimation," to appear in IEEE Transactions on Signal Processing

[6] M. Haardt and J.A. Nossek, "Unitary ESPRIT- how to obtain increased estimation accuracy with a reduced computational burden," IEEE Transactions on Signal Processing, vol.43, no.5, pp.1232-1242, May. 1995

[7] O. Besson, P. Stoica, "Two subspace-based methods for frequency estimation of sinusoidal signals with random amplitude," IEE Proceedings on Radar, Sonar and Navigation, vol.144, no.4, pp.169 - 176, Aug. 1997

[8] G. H. Golub and C. F. Van Loan, Matrix Computations, 2nd edition, Baltimore, MD: The Jhons Hopkins University Press, 1996 , 


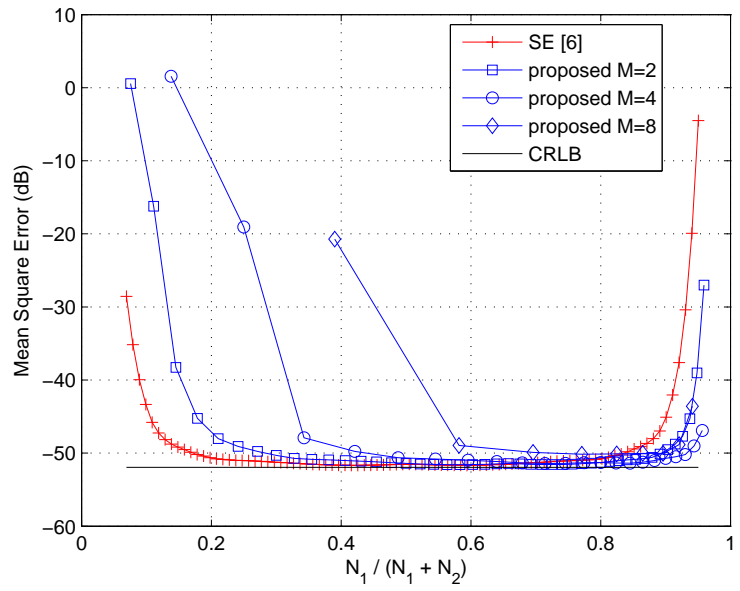

Fig. 1. Average mean square frequency error versus $\frac{N_{1}}{N_{1}+N_{2}}$.

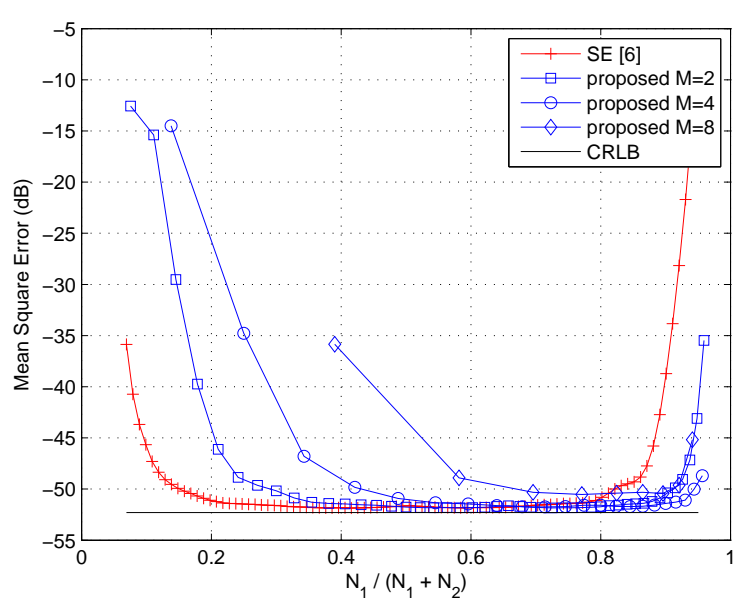

Fig. 2. Average mean square damping factor error versus $\frac{N_{1}}{N_{1}+N_{2}}$.

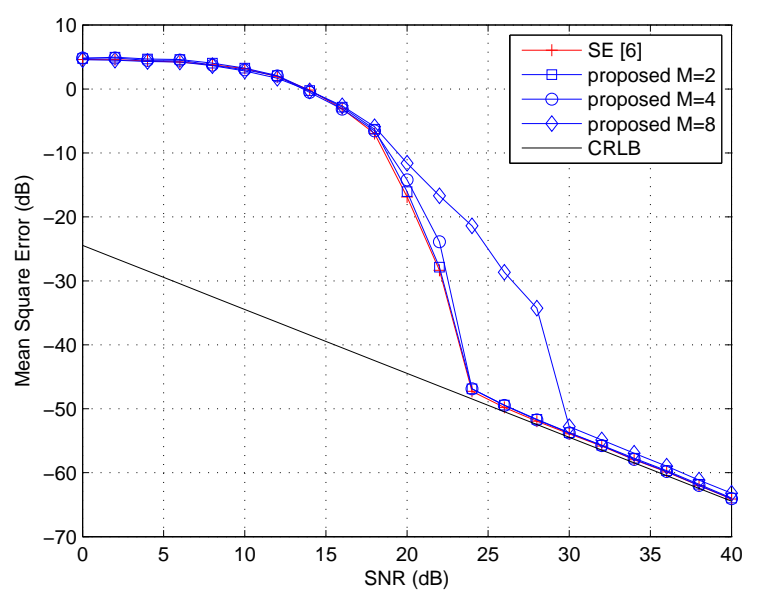

Fig. 3. Average mean square frequency error versus SNR.

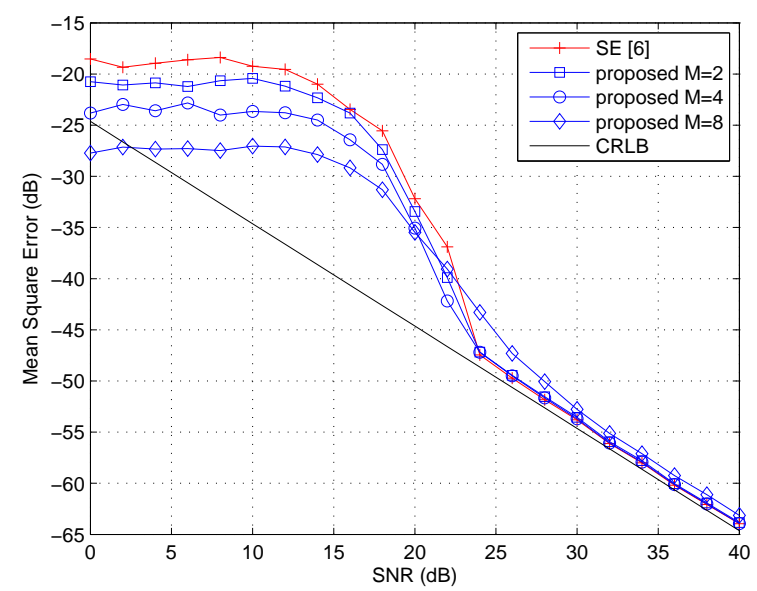

Fig. 4. Average mean square damping factor error versus SNR.

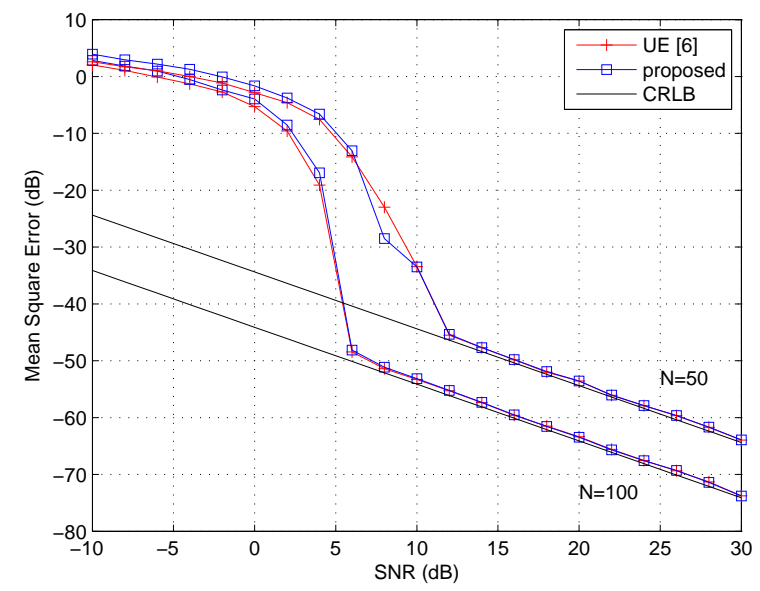

Fig. 5. Average mean square frequency error versus SNR for different $\mathrm{N}$.

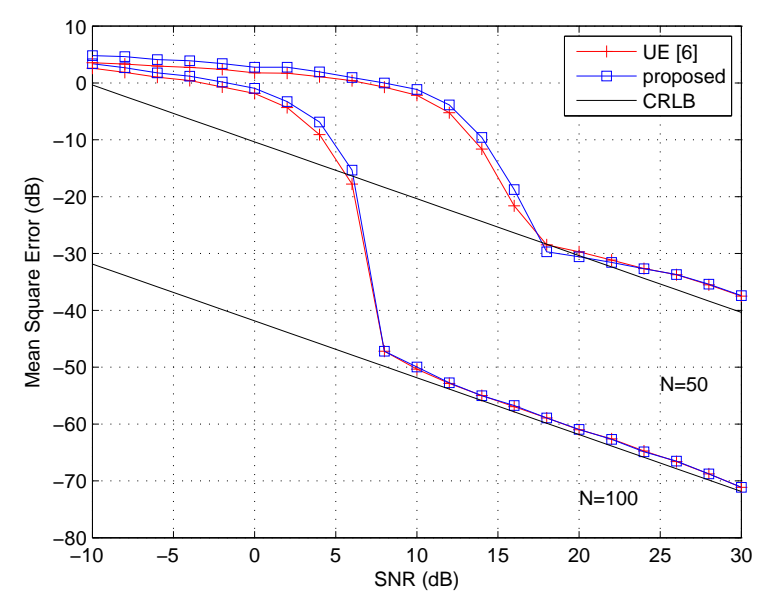

Fig. 6. Average mean square damping factor error versus SNR with small frequency seperations. 